\title{
A Space Odyssey: Experimental Manipulation of Threat Perception and Anxiety-Related Interpretation Bias in Children
}

\author{
Peter Muris $\cdot$ Jorg Huijding $\cdot$ Birgit Mayer $\cdot$ Marjolein Hameetman
}

Published online: 30 April 2008

(C) The Author(s) 2008

\begin{abstract}
This study provides a first test of an experimental method, the "space odyssey" paradigm, that was designed to manipulate interpretation bias in children. Seventy nonclinical children aged 8-12 years first completed a standardized anxiety questionnaire. Following this, they completed the space odyssey paradigm to induce either a negative or a positive interpretation bias. After this stage of interpretation training, children were presented with a series of ambiguous vignettes for which they had to rate perceived levels of threat as an index of interpretation bias. Results indicated that the space odyssey paradigm was successful in training interpretations: children in the negative training condition quickly learned to choose negative outcomes, while children in the positive training condition rapidly learned to select positive outcomes. Most importantly, children's subsequent threat perception scores for the ambiguous vignettes were affected by the manipulation. That is, children in the negative training condition perceived more threat than children in the positive training condition. Interestingly, the effects of training were most pronounced in high anxious children. Directions for future research with this paradigm are briefly discussed.
\end{abstract}

Keywords Children · Anxiety - Interpretation bias - Interpretation training · Experimental manipulation

\section{Introduction}

According to Kendall's [1] cognitive theory, pathological manifestations of childhood anxiety result from the chronic overactivity of schemas organized around themes of vulnerability and danger. These overactive schemas are assumed to focus processing resources chronically on threat-relevant information and manifest themselves in so-called cognitive distortions. These distortions pertain to biased cognitive processes that yield erroneous and

P. Muris $(\varangle) \cdot$ J. Huijding $\cdot$ B. Mayer $\cdot$ M. Hameetman Institute of Psychology, Erasmus University Rotterdam, Burgemeester Oudlaan 50, Suite T13-37, P.O. Box 1738, 3000 DR Rotterdam, The Netherlands e-mail: muris@fsw.eur.nl 
maladaptive thoughts and behaviors [2,3]. One example of such an anxiety-related cognitive distortion is interpretation bias, which refers to the tendency to attach a threatening meaning to ambiguous stimuli. Interpretation bias might explain why anxious children so readily evaluate fairly benign situations as dangerous.

Various studies have demonstrated that interpretation bias is present in anxious youths. For example, Bell-Dolan [4] presented 9- to 11-year-old children who were high or low on trait anxiety, with a series of videotaped peer-interaction scenarios displaying peer behaviour that was either hostile, non-hostile, or ambiguous. Results showed that the highanxious children were equally accurate in identifying hostile intent in peer interactions as their low-anxious counterparts. However, high-anxious children more frequently interpreted the non-hostile and ambiguous scenarios as threatening. In another study of Barrett et al. [5], anxiety disordered children, children with oppositional defiant disorder, and normal controls (all aged between 7 and 14 years) were presented with vignettes of ambiguous situations and asked about what was happening in each situation. Following this, children were given two possible neutral outcomes and two possible threatening outcomes and were asked to indicate which outcome was most likely to occur. Results showed that both anxious and oppositional children more frequently perceived ambiguous situations as threatening than did normal controls, with anxious children more often choosing avoidant outcomes and oppositional children more frequently choosing aggressive outcomes [see also 6,7]. In addition, a series of studies conducted by Muris and colleagues [8-10] has shown that anxious children only need very minor threat cues before interpreting ambiguous vignettes as threatening. Adopting a different experimental method, Hadwin et al. [11] confronted 7- to 9-year-old children with homophones (i.e., words that sound the same but have different meanings) that either had a neutral or a threatening meaning (e.g., dye versus die). The results showed that children's anxiety levels were positively associated with the tendency to interpret the homophones as threatening. A comparable approach was followed by Taghavi et al. [12] who presented 9to 16-year-old youths with homographs (i.e., words that are spelled the same but have different meanings; e.g., tear) that were printed on cards. Participants were asked to construct a sentence that included the homograph. The results of this study indicated that anxious children and adolescents produced significantly more sentences that were consistent with a threatening homograph interpretation than did the control children. All these studies provide a demonstration of anxious children's inclination to perceive the external world as dangerous and illustrate how easily these youths become entangled in anxious feelings and thought processes.

Few investigations have examined the origins of interpretation bias in child populations. While it is generally assumed that inherited temperament or personality characteristics such as neuroticism for a large part determine this type of cognitive distortion [2, 3], there is some recent evidence indicating that environmental influences are also involved. For example, Creswell et al. [13] employed an ambiguous scenario task to measure interpretation bias in a mixed sample of clinically anxious and non-clinical children (aged 7-15 years) and their mothers. The results showed that children's and mothers' interpretation bias scores were positively correlated, suggesting that "children's information processing style may result from internalisation of parental perception of threat" [13, p. 1379; see also 14]. In a subsequent longitudinal study, Creswell et al. [15] examined whether mothers' anxious cognitions were predictive of children's interpretation bias over time (i.e., a period of 6 months). Results indeed revealed the hypothesised prospective link, which provides further support for the idea that family influences are involved in the formation of interpretation bias [16]. 
While the above described studies indicate that interpretation bias is passed on from parents (i.e. mothers) to their children, it remains unclear what mechanism is responsible for the transfer of this cognitive bias. One possibility is that biased interpretation is installed by anxious parents who, due to their own interpretation bias, frequently provide negative feedback about ambiguous stimuli and situations to their children. In line with this idea, recent evidence from the adult literature has shown that a threat-related interpretation bias can be induced by training participants to generate negative outcomes for ambiguous stimuli. For example, Mathews and Mackintosh [17] confronted their participants with brief ambiguous vignettes, and instructed them to attach either a positive or a negative interpretation to these vignettes by completing one of two word fragments. Participants received feedback (i.e., a correct or a wrong answer message) about their responses in such way that one group was learned to disambiguate the vignettes in a threatening way, whereas the other group was taught to resolve the vignettes in a benign way. Results demonstrated that the training was successful: participants in the negative training group responded significantly faster to negatively toned fragments, while participants in the positive training group responded more quickly to positively toned fragments. Most importantly, a subsequent testing stage during which participants had to interpret a new set of ambiguous vignettes, revealed that their interpretation style was indeed changed. That is, participants in the negative training group more frequently chose negative interpretations, whereas participants in the positive training group more often endorsed positive interpretations. These and other findings suggest that interpretation bias can be successfully manipulated by learning experiences [18-23].

Thus far, all studies on the experimental training of interpretation bias have been conducted with adults. Meanwhile this research might provide valuable insight in the origins of this type of cognitive distortion (see supra) and allied anxiety symptoms in children. Of course the challenge is to develop an experimental paradigm that is suitable for manipulating interpretation bias in young people, which is acceptable from an ethical point of view as well. With this in mind, we developed the "space odyssey" paradigm, which essentially is a computer game of an imaginary journey to an unknown planet. During the first part of the game, children get acquainted with the unknown living conditions on the new planet: they are presented with brief scenarios describing unknown situations. For each situation, children have to choose between a negative (i.e., mildly threatening) or a positive outcome. Children continuously receive feedback on the correctness of their choices. In one half of the children the choice of negative outcomes is reinforced (i.e., negative training group), whereas in the other half the choice of positive outcomes is reinforced (i.e., positive training group). The second part of the game actually is an interpretation bias task: children are asked to imagine that they still live on the new planet and confronted with ambiguous scenarios describing everyday situations that could also occur on earth (e.g., going to school, encountering unfamiliar people). The main task of the children is to rate the level of threat associated with each of these situations. The present article describes a first attempt to manipulate interpretation bias in children using this "space odyssey" paradigm. As this study provides a test of the suitability of this training method, it was conducted in a sample of non-clinical children. However, to explore whether the manipulation has differential impact on high- and low-anxious children, we also measured levels of anxiety disorders symptoms. It was hypothesized that children in the negative training group would display higher levels of threat perception than children in the positive training group. In addition, it was expected that the negative training would have more impact on relatively high-anxious children and thus results in a stronger cognitive bias than in low-anxious children. 


\section{Method}

Participants

The parents of 80 children from a regular primary school in Dordrecht, the Netherlands, were approached by mail with the request to participate in the current study. Most parents $(87.5 \%)$ responded favourable to this invitation and completed and signed the informed consent form. Six parents did not allow their child to participate, whereas four other parents did not respond to our mailing at all. The 70 participating children (34 boys and 36 girls) had a mean age of 10.03 years $(S D=0.88$; range $8-12$ years). The research project was officially approved by the Ethical Committee of Psychological Research of Erasmus University Rotterdam. Due to school constraints, no exact information about the socioeconomic background of the youths was available. However, $14.3 \%$ of the children was non-Caucasian, which in the Netherlands is generally indicative for a lower socioeconomic status.

\section{Questionnaire}

The Screen for Child Anxiety Related Emotional Disorders (SCARED) is a self-report questionnaire for measuring anxiety disorder symptoms in children and adolescents [24, 25]. The scale consists of 41 items that can be allocated to five subscales. Four of the subscales parallel anxiety disorders as described in the Diagnostic and Statistical Manual of Mental Disorders [26], namely panic/somatic (e.g., "When frightened, my heart beats fast"), generalized anxiety (e.g., "I am a worrier"), separation anxiety (e.g., "I don't like being away from my family"), and social phobia (e.g., "I don't like to be with unfamiliar people"). The fifth subscale is school phobia (e.g., "I am scared to go to school") which represents a common anxiety problem in youths. Children are asked to rate the frequency with which they experience each symptom using three-point scales $(0=$ almost never, $1=$ sometimes, and $2=$ often). SCARED total and subscale scores are obtained by summing relevant items. Research has demonstrated that the SCARED has good internal consistency, test-retest reliability, and validity, and this conclusion appears true for clinical as well as non-clinical samples of youths [24, 25, 27].

\section{The "Space Odyssey" Paradigm}

This paradigm was specifically construed for the purpose of the present study. Essentially this paradigm involves a computer task that is programmed in E-prime (Version 1.0) [28]. Prior to the real task, children were given the following general instruction, which was presented to them on the computer screen: "It is the year 2010. You and your parents are astronauts who travel through space. You are searching for a new planet where people can live. After a long journey, you discover a big planet. You decide to land on the planet and to explore it. When the spacecraft has landed, you discover that the planet resembles the earth in many ways, but there are also a lot of things that you do not know". The computer task consisted of two stages: (1) a training stage during which either a negative or a positive interpretation bias was induced, and (2) a testing stage that was employed to measure interpretation bias.

The training stage consisted of 30 trials. In every trial children were presented with a short scenario describing an unknown situation that might happen on the unknown planet. 
Each scenario was followed by two options, one describing a positive outcome, the other describing a negative outcome. For example, the scenario "On the street, you encounter a spaceman. He has a sort of toy handgun and he fires at you..." was followed by (A) "You are laughing: it is a water pistol and the weather is fine anyway", and (B) "Oops, this hurts! The pistol produces a red beam which burns your skin!" (for further examples, see Appendix A). Children were instructed to read the scenario and the two possible outcomes and then to choose one of the outcomes by pressing the A or B button on the keyboard of the computer. Directly after pressing one of the buttons, the scenario and outcomes disappeared from the screen to be replaced by either the word "Good" or "Wrong" thereby proving the children with feedback on their choice. In the negative training condition, the choice of a negative outcome was always followed by "Good!" and the choice of a positive outcome was always followed by "Wrong!". In the positive training condition, these outcome-feedback sequences were reversed. Thus, in the negative training condition the choice of a negative outcome was reinforced, whereas in the positive training condition the choice of a positive outcome was reinforced.

The testing stage consisted of ambiguous vignettes that have been employed in previous research to measure interpretation bias in children [10, 29]. Nine vignettes were used, all describing ambiguous situations that children may encounter in daily life (i.e., giving an oral report in front of the class, going to the sporting club for the first time, staying home alone in the evening, not seeing one's mother in a large shopping mall, staying with a friend while parents are on vacation, meeting unknown people who have come to visit mother, having to take an important test at school, encountering unfamiliar men in a big city, driving a bike on a busy street; for an example see Appendix B). Each vignette was construed in such way that children were still given the idea that they were confronted with these (everyday) situations during their space journey. This was achieved by including the word "space" in the first sentence of each vignette (e.g., "school" was described as "space-school", "city" as "space-city", "bike" as "space-bike"). Children started this stage by reading the following instruction on the computer screen: "In a moment, you are going to read a number of brief stories. Some stories are scary: this means that these stories will have a bad ending. Some stories are not scary: this means that these stories will have a good ending (note: in fact all vignettes were ambiguous). Stories will be presented to you sentence-by-sentence, and after each sentence you have to make a prediction on how scary that story is going to become by pressing a figure on the keyboard ranging between 1, which means not at all scary and 7, which indicates very scary" (the pertinent buttons of the keyboard were highlighted in order to facilitate children's responses). As there were nine vignettes that were each composed of 5 sentences, there were 45 responses. As a result, scores on this test varied between 45 and 315, with higher scores reflecting higher levels of threat perception (i.e., negative interpretation bias).

\section{Procedure}

After obtaining informed consent from the parents, children first completed the SCARED during a classroom session. The teacher and the research assistant were present during this assessment in order to ensure confidential responding and to provide assistance if necessary. Within two weeks after completion of the questionnaire, children were tested individually with the computer task by a trained female research assistant in a separate room at school. Children were randomly allocated to either the negative training ( $n=34$, 15 boys and 19 girls, mean age $=10.12$ years, $S D=0.81$ ) or the positive training 
condition $(n=36,19$ boys and 17 girls, mean age $=9.94, S D=0.95)$. The assistant was blind to children's questionnaire scores. She first briefly introduced herself, comforted the child, and then started the E-prime program on the computer (a MacBook). The assistant remained present during the full procedure: she read the instructions together with the child, but kept herself apart during the training and testing stage of the experiment.

\section{Statistical Analysis}

Analyses of variance (ANOVAs) were employed to examine whether the experimental training was successful and to evaluate differences in threat perception of the ambiguous vignettes (i.e., negative interpretation bias) between children in the negative and positive training groups. As it is known from the literature that anxiety is an important correlate of this type of cognitive bias [2,3], this variable was included as a covariate in the latter analysis. Further, given the fairly wide age range of the children included in the present study and the fact that girls generally possess a greater vulnerability to display anxiety and related phenomena [30], age and gender were also entered as covariates.

\section{Results}

Reliability of Measures

Mean scores on the SCARED and the ambiguous vignettes test were $17.70(S D=9.82)$ and 126.09 ( $S D=50.56)$, respectively. The SCARED total score had an internal consistency coefficient (Cronbach's alpha) of .88, which indicates that this anxiety scale was highly reliable. Further, scores obtained with the vignette task (testing stage) were also reliable in terms of internal consistency, with Cronbach's alphas of .95 and .96 in negative and positive training groups, respectively. Thus, the main instruments that were used in this study proved to be highly reliable.

\section{Effects During Training Stage: Did Children Learn the Rule?}

In order to evaluate whether children actually learned to choose either the negative or the positive outcomes in a consistent way during the training stage, children's responses were first divided into 6 blocks of 5 trials each. Then children's average percentages of correct responses were subjected to a 2 (training: negative vs. positive) $\times 6$ (blocks) repeated measure ANOVA. This analysis yielded a significant main effect of blocks $[F(5$, $340)=34.29, p<.001$, partial $\left.\eta^{2}=.34\right]$. Post-hoc tests indicated that the percentage of correct trials significantly increased from block 1 to block $2[t(69)=7.58, p<.001]$, after which no further between-block differences were observed. As can be seen in the top panel of Fig. 1, in both the negative and positive training condition children quickly learned the 'interpretation rule' and then consistently tended to choose the congruent outcome. In addition, the interaction effect of training and block also reached statistical significance $\left[F(5,340)=3.01, p<.05\right.$, partial $\left.\eta^{2}=.04\right]$ : children appeared to learn the interpretation rule more quickly in the positive training condition than in the negative training condition. A closer inspection of the first two blocks (see the bottom panel of Fig. 1) indicated that differences in correct responses between the negative and positive training group were only significant during the first two trials of the experiment [both $t(68) \mathrm{s} \geq 3.48, p \mathrm{~s}<.001$ ] 


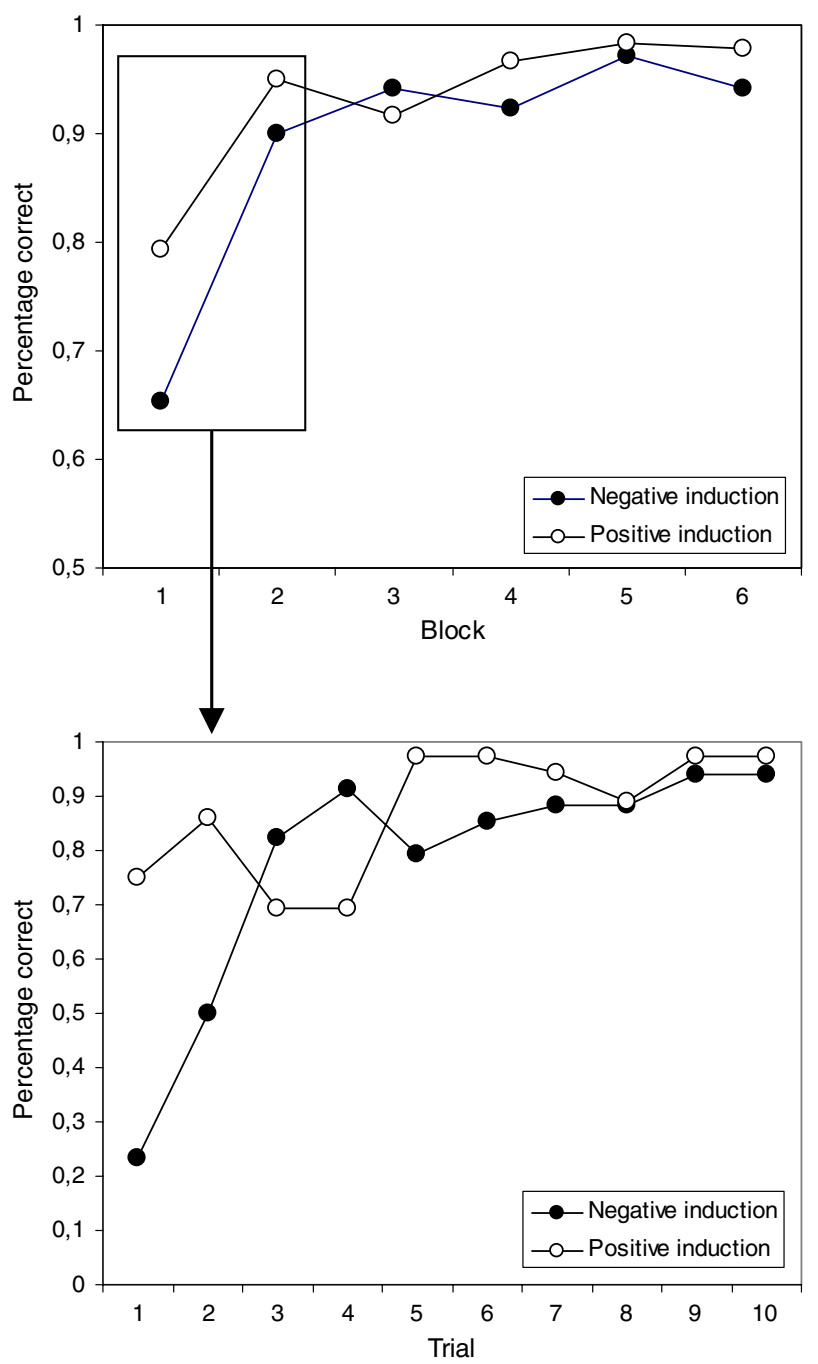

Fig. 1 Percentage of children's correct responses during the full training stage (consisting of 6 blocks of 5 trials each; upper graph) and during the first two blocks (consisting of 5 trials each; lower graph) in which either a negative or a positive interpretation bias was trained

Altogether, the training stage appeared to be successful: children in the negative training group learned to choose the negative outcomes, whereas children in the positive training group learned to select the positive outcomes.

Effects During Testing Stage: Was Interpretation Bias Successfully Manipulated?

To examine the effects of the training stage on children's interpretation bias, an ANOVA was carried out to compare the threat perception scores of children in both training conditions, which included SCARED anxiety symptoms, gender, and age as covariates. Most importantly, the crucial main effect of training was significant $[F(1,65)=13.43, p<.01$, 
partial $\left.\eta^{2}=.17\right]$ : adjusted mean threat perception scores were significantly higher in the negative $(M=144.62, S E=7.01)$ than in the positive training group $(M=108.58$, $S E=6.81)$. The effects of the covariates gender $[F(1,65)=6.72, p<.05$, partial $\left.\eta^{2}=.09\right]$ and SCARED anxiety symptoms ${ }^{1}\left[F(1,65)=16.02, p<.001\right.$, partial $\left.\eta^{2}=.20\right]$ were also significant.

To examine the effect of gender more precisely, a 2 (training) $\times 2$ (gender) ANOVA with SCARED anxiety symptoms and age as covariates was carried out. The main effect of gender remained significant $\left[F(1,64)=6.62, p<.05\right.$, partial $\left.\eta^{2}=.09\right]$, but there was no interaction effect of gender and training $[F(1,64)=.04]$. Inspection of the adjusted mean threat perception scores indicated that girls clearly displayed higher threat perception scores than did boys, means being 139.19 ( $S E=6.95)$ and 113.07 ( $S E=7.18)$, respectively.

To investigate the effect of anxiety in more detail, a gender-specific mean split procedure was used to divide the sample in high-anxious ( $n=34,18$ boys and 16 girls, mean age $=9.94, S D=0.86)$ and low-anxious children $(n=36,16$ boys and 20 girls, mean age $=10.11, S D=0.89$ ). A 2 (training) $\times 2$ (anxiety group) ANOVA with age and gender as covariates performed on children's threat perception scores revealed significant main effects of training $\left[F(1,64)=16.62, p<.001\right.$, partial $\left.\eta^{2}=.21\right]$ and anxiety group $\left[F(1,64)=9.32, p<.01\right.$, partial $\left.\eta^{2}=.13\right]$. Interestingly, the interaction of training and anxiety group was also significant $\left[F(1,64)=4.11, p<.05\right.$, partial $\left.\eta^{2}=.06\right]$. Post-hoc comparisons indicated that although the negative training generally seemed to have enhanced children's threat perception scores, this effect only reached statistical significance in the high-anxious group $[t(32)=4.34, p<.001]$ (see Fig. 2).

\section{Discussion}

Various studies with adult populations have demonstrated that interpretation biases can be experimentally manipulated [17-23]. The present article describes a first attempt to manipulate interpretation bias in children, using a newly developed experimental procedure, the space odyssey paradigm. Non-clinical children aged 8-12 years first underwent a training stage which had the purpose to induce either a negative or a positive interpretation bias. To test whether the paradigm was successful in inducing the intended bias, children were subsequently presented with a series of ambiguous vignettes for which threat perceptions were assessed. Results showed that the training stage was successful: children in the negative training condition quickly learned to choose negative outcomes for the unknown scenes, whereas children in the positive training condition rapidly learned to select positive outcomes. Most importantly, children's subsequent threat perception scores were affected by this manipulation. That is, children in the negative training condition displayed higher threat perception scores than children in the positive training condition. Moreover, an additional analysis indicated that this pattern of results was particularly pronounced for children who already exhibited high levels of anxiety symptoms. The latter finding is interesting because it suggests that more vulnerable (i.e., already more anxious) children were especially affected by our experimental manipulation.

Altogether the results produced by our "space odyssey" paradigm are interesting in that they show that it is possible to change interpretation bias in child populations.

\footnotetext{
1 The correlations between the SCARED and ambiguous vignette test scores were $.40(p<.001)$ in the total group, $.60(p<.001)$ in the negative training group, and $.21(p=.22)$ in the positive training group.
} 
Fig. 2 Mean threat perception scores (error bars represent standard errors) of high-anxious and low-anxious children in the negative and positive interpretation bias training groups

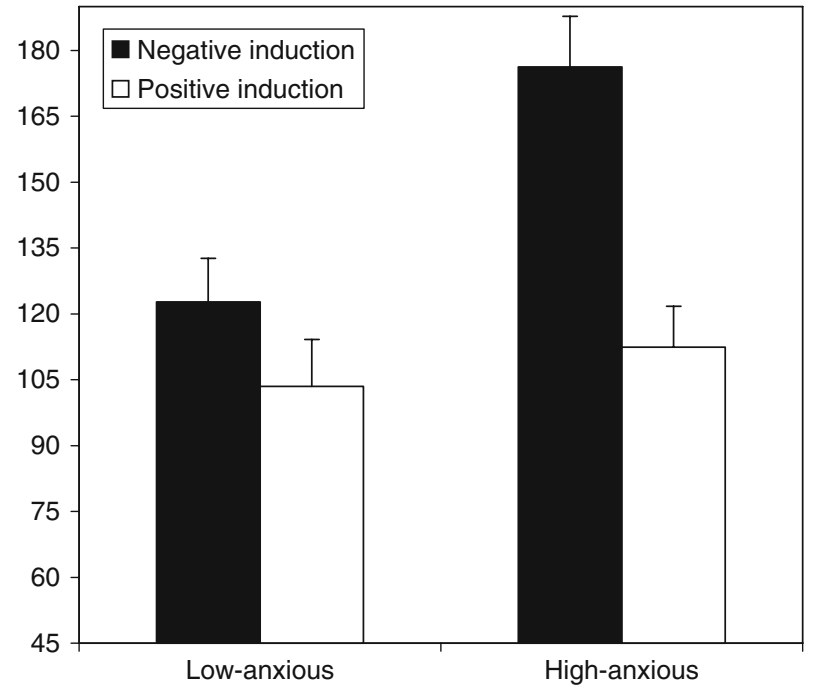

Nevertheless, there are a number of important issues that need to be resolved in future research. First of all, as the present study did not include a baseline assessment, we were not able to study the change in interpretation bias as a result of the experimental manipulation. A pre- to post-test design will be necessary to definitely demonstrate that a negative training indeed enhances children's threat perception scores. Further, such a design may reveal whether a positive training is able to reduce this type of cognitive bias, especially in high-anxious children. Second, we only investigated whether the experimental procedure had an effect on children's cognitive style. Thus, it remains to be seen whether this experimental paradigm also produces an effect on children's anxiety levels. Although previous research has shown that this seems to be the case in adults [17], it remains to be seen whether such effects can also be demonstrated in child populations. Third, during the training stage, a $100 \%$ contingency was employed to manipulate the cognitive bias. While this seemed to be a "safe" strategy for a first study to investigate the manipulation of interpretation bias in children, it is clear that future studies should investigate the effects at varying contingencies. Fourth and finally, the possibility cannot be ruled out that the current results are at least in part explained by experimental demand. That is, children rapidly learned the interpretation rule during the training stage and then continued to employ this rule during the rest of the experiment. New studies could tackle this problem by employing more 'covert' methods for assessing interpretation bias (e.g., by means of homographs, homophones).

This study also yielded a number of additional findings that deserve some brief comment. To begin with, in keeping with previous research [2, 3], a clear link between children's anxiety symptoms and interpretation bias scores was observed. According to cognitive theory [31], cognitive distortions underlie anxiety problems in youths, but in fact few studies have actually addressed this issue. Future research with the "space odyssey" paradigm as well as studies examining the prospective relation between interpretation bias and anxiety over longer time periods are needed to address this important topic [32]. Further, there was a significant gender difference with respect to interpretation bias, with girls displaying a stronger tendency towards this cognitive distortion than boys. Although this issue has been somewhat neglected in previous research, this result fits nicely with the 
observation that girls seem to be more anxiety-prone than boys [33, 34]. Finally, the results of the training stage seem to suggest that the positive training worked faster than the negative training. That is, during the first trials of the experiment, children in the positive training condition provided more correct responses than children in the negative training condition. This finding was probably due to the fact that in this sample of non-clinical children there was an initial tendency to choose the positive outcomes for the unknown scenarios. For children in the positive training condition this inclination was immediately confirmed. However, for children in the negative training condition this initial tendency towards the positive first had to be corrected.

As already pointed out, the present study suffered from various methodological limitations that at the same time provide a useful guide for future studies. Of course, another shortcoming pertains to the imaginary character of our "space odyssey" paradigm, which was purely governed by ethical considerations. Nevertheless, the paradigm provides an experimental method that can be used to learn more about the origins of interpretation bias in children. An interesting venue for future studies would be to try to adapt the paradigm so that it becomes suitable for changing this type of cognitive distortions in real life. Recent studies with adults have demonstrated that the training of a benign interpretation bias was effective in reducing trait anxiety [35] and social anxiety [36], and it would be interesting to study whether similar positive results can be achieved in high-anxious youths.

\section{Summary}

Interpretation bias refers to a cognitive distortion that can be defined as the tendency to disproportionately perceive threat in ambiguous situations, and is thought to play a role in the continuation of anxiety problems [2,3]. Previous work in adults has shown that interpretation bias can be experimentally induced [17-23], which opens up the possibility to explore the origins of this type of cognitive distortion and to further examine its role in the development of anxiety. The present article describes an experimental paradigm (i.e., the "space odyssey" paradigm) that is suitable for manipulating interpretation bias in child populations. The data indicate that by means of the paradigm children's subsequent threat perception scores for the ambiguous vignettes can be successfully manipulated. Future research should further investigate the applicability of the paradigm to change interpretation bias and anxiety in youth.

Acknowledgment Children, parents, and staff of primary school "De Keerkring" in Dordrecht, The Netherlands, are thanked for their participation in this study.

Open Access This article is distributed under the terms of the Creative Commons Attribution Noncommercial License which permits any noncommercial use, distribution, and reproduction in any medium, provided the original author(s) and source are credited.

\section{Appendix A}

Further examples of the brief scenarios that were used to induce interpretation bias.

1. In the wood, you see fruit hanging in a big tree. The fruit looks like apples. You take one of them and you have a bite... 

A. Wow, this fruit tastes very well.
B. Yuck! This fruit tastes very nasty.

2. On the plaza you find a beautiful silver ball. You pick it up because you want to play with it....
A. The ball is nice and bounces very well. You take it with you to show it to a friend.
B. Ouch! That hurts. The ball gives a painful electric shock. You are crying and run to your home.

3. In the garden you see funny, little green birds in a tree. You go inside to get some bread that you can feed to them...
A. That is funny. The birds come really close and one of them even eats the bread from your hand.
B. Oops, this was not a good idea. The birds are really wild and begin to peck you in your arms and legs.

\section{Appendix B}

Example of a vignette that was employed to measure threat perception and interpretation bias.

1. You have decided to join a space-sporting club.

2. You are for the first time in the changing-room of the sporting club.

3. There you see a group of children waiting in a row.

4. You don't know any of them.

5. They all look at you.

\section{References}

1. Kendall PC (1985) Toward a cognitive-behavioral model of child psychopathology and a critique of related interventions. J Abnorm Child Psychol 13:357-372

2. Muris P, Field A (2008) Distorted cognition and pathological anxiety in children and adolescents. Cogn Emot 22:395-421

3. Vasey MW, MacLeod C (2001) Information-processing factors in childhood anxiety: a review and developmental perspective. In: Vasey MW, Dadds MR (eds) The developmental psychopathology of anxiety. Oxford University Press, New York, pp 253-277

4. Bell-Dolan DJ (1995) Social cue interpretation of anxious children. J Clin Child Psychol 24:1-10

5. Barrett PM, Rapee RM, Dadds MR, Ryan SM (1996) Family enhancement of cognitive style in anxious and aggressive children. J Abnorm Child Psychol 24:187-203

6. Bögels SM, Zigterman D (2000) Dysfunctional cognitions in children with social phobia, separation anxiety disorder, and generalized anxiety disorder. J Abnorm Child Psychol 28:205-211

7. Chorpita BF, Albano AM, Barlow DH (1996) Cognitive processing in children: relationship to anxiety and family influences. J Clin Child Psychol 25:170-176

8. Muris P, Luermans J, Merckelbach H, Mayer B (2000) "Danger is lurking everywhere". The relationship between anxiety and threat perception abnormalities in normal children. J Behav Ther Exp Psychiatry 31:123-136

9. Muris P, Merckelbach H, Damsma E (2000) Threat perception bias in nonreferred socially anxious children. J Clin Child Psychol 29:348-359

10. Muris P, Rapee R, Meesters C, Schouten E, Geers M (2003) Threat perception abnormalities in children: the role of anxiety disorders symptoms, chronic anxiety, and state anxiety. J Anxiety Disord $17: 271-287$ 
11. Hadwin J, Frost S, French CC, Richards A (1997) Cognitive processing and trait anxiety in typically developing children: evidence for interpretation bias. J Abnorm Psychol 106:486-490

12. Taghavi MR, Moradi AR, Neshat-Doost HT, Yule W, Dalgleish T (2000) Interpretation of ambiguous emotional information in clinically anxious children and adolescents. Cogn Emot 14:809-822

13. Creswell C, Schiering CA, Rapee RM (2005) Threat interpretation in anxious children and their mothers: comparison with nonclinical children and the effects of treatment. Behav Res Ther 43: 1375-1381

14. Creswell C, O'Connor TG (2006) 'Anxious cognitions' in children: an exploration of associations and mediators. Br J Dev Psychol 24:761-766

15. Creswell C, O'Connor TG, Brewin CR (2006) A longitudinal investigation of maternal and child 'anxious cognitions'. Cognit Ther Res 30:135-147

16. Hadwin J, Garner M, Perez-Olivas G (2006) The development of information processing biases in childhood anxiety: a review and exploration of its origins in parenting. Clin Psychol Rev 26:876-894

17. Mathews A, Mackintosh B (2000) Induced emotional interpretation bias and anxiety. J Abnorm Psychol 109:602-615

18. Grey S, Mathews A (2000) Effects of training on interpretation of emotional ambiguity. Q J Exp Psychol A 53:1143-1162

19. Mathews A, MacLeod C (2002) Induced processing biases have causal effects on anxiety. Cogn Emot $16: 331-354$

20. Salemink E, Van den Hout MA, Kindt M (2007) Trained interpretive bias and anxiety. Behav Res Ther 45:329-340

21. Salemink E, Van den Hout MA, Kindt M (2007) Trained interpretive bias: validity and effects on anxiety. J Behav Ther Exp Psychiatry 38:212-224

22. Wilson E, MacLeod C, Mathews A, Rutherford EM (2006) The causal role of interpretative bias in anxiety reactivity. J Abnorm Psychol 115:103-111

23. Yiend J, Mackintosh B, Mathews A (2005) Enduring consequences of experimentally induced biases in interpretation. Behav Res Ther 43:779-797

24. Birmaher B, Brent D, Chiappetta L, Bridge J, Monga S, Baugher M (1999) Psychometric properties of the Screen for Child Anxiety Related Emotional Disorders (SCARED): a replication study. J Am Acad Child Adolesc Psychiatry 38:1230-1236

25. Birmaher B, Khetarpal S, Brent D, Cully M, Balach L, Kaufman J, McKenzie Neer S (1997) The Screen for Child Anxiety Related Emotional Disorders (SCARED): scale construction and psychometric characteristics. J Am Acad Child Adolesc Psychiatry 36:545-553

26. American Psychiatric Association (2000) Diagnostic and statistical manual of mental disorders, 4th edn-text revision (DSM-IV-TR). American Psychiatric Association, Washington, DC

27. Muris P, Merckelbach H, Ollendick TH, King NJ, Bogie N (2002) Three traditional and three new childhood anxiety questionnaires: their reliability and validity in a normal adolescent sample. Behav Res Ther 40:753-772

28. Schneider W, Eschman A, Zuccolotto A (2002) E-prime user guide. Psychology Software Tools, Pittsburgh, PA

29. Muris P, Kindt M, Bögels S, Merckelbach H, Gadet B, Moulaert V (2000) Anxiety and threat perception abnormalities in normal children. J Psychopathol Behav Assess 22:183-199

30. Ollendick TH, Yang B, Dong Q, Xia Y, Lin L (1995) Perceptions of fear in other children and adolescents: the role of gender and friendship status. J Abnorm Child Psychol 23:439-452

31. Beck AT, Emery G, Greenberg RL (1985) Anxiety disorders and phobias: a cognitive perspective. Basic Books, New York, NY

32. Muris P, Jacques P, Mayer B (2004) The stability of threat perception abnormalities and anxiety disorder symptoms in non-clinical children. Child Psychiatry Hum Dev 34:251-265

33. Craske MG (2003) Origins of phobias and anxiety disorders. Why more women than men? Elsevier, Oxford, UK

34. Lewinsohn PM, Gotlib IH, Lewinsohn M, Seeley JR, Allen NB (1998) Gender differences in anxiety disorders and anxiety symptoms in adolescents. J Abnorm Psychol 107:109-117

35. Mathews A, Ridgeway V, Cook E, Yiend J (2007) Inducing a benign interpretational bias reduces trait anxiety. J Behav Ther Exp Psychiatry 38:225-236

36. Murphy R, Hirsch CR, Mathews A, Smith K, Clark DM (2007) Facilitating a benign interpretation bias in a high socially anxious population. Behav Res Ther 45:1517-1529 